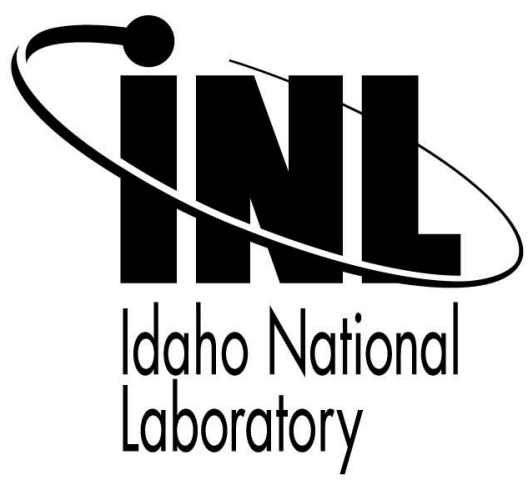

\title{
Unique Method for Generating Design Earthquake Time Histories
}

\section{ASME Pressure Vessels and Piping Division Conference}

\author{
R. E. Spears
}

July 2008

This is a preprint of a paper intended for publication in a journal or proceedings. Since changes may be made before publication, this preprint should not be cited or reproduced without permission of the author. This document was prepared as an account of work sponsored by an agency of the United States Government. Neither the United States Government nor any agency thereof, or any of their employees, makes any warranty, expressed or implied, or assumes any legal liability or responsibility for any third party's use, or the results of such use, of any information, apparatus, product or process disclosed in this report, or represents that its use by such third party would not infringe privately owned rights. The views expressed in this paper are not necessarily those of the United States Government or the sponsoring agency. 
PVP2008-61243

\title{
UNIQUE METHOD FOR GENERATING DESIGN EARTHQUAKE TIME HISTORIES
}

\author{
R. E. Spears, Ph.D. \\ Senior Research Engineer \\ Idaho National Laboratory \\ Tel: 208-526-4109 \\ Fax: 208-526-4311 \\ E-Mail: Robert.Spears@inl.gov
}

\begin{abstract}
A method has been developed which takes a seed earthquake time history and modifies it to produce given design response spectra. It is a multi-step process with an initial scaling step and then multiple refinement steps. It is unique in the fact that both the acceleration and displacement response spectra are considered when performing the fit (which primarily improves the low frequency acceleration response spectrum accuracy). Additionally, no matrix inversion is needed. The features include encouraging the code acceleration, velocity, and displacement ratios and attempting to fit the pseudo velocity response spectrum. Also, "smoothing" is done to transition the modified time history to the seed time history at its start and end. This is done in the time history regions below a cumulative energy of $5 \%$ and above a cumulative energy of $95 \%$. Finally, the modified acceleration, velocity, and displacement time histories are adjusted to start and end with an amplitude of zero (using Fourier transform techniques for integration).
\end{abstract}

\section{INTRODUCTION}

Time history analysis is an important technique for structural seismic analysis especially when the evaluated structural response is nonlinear. To perform such an analysis, a representative earthquake time history (or seed time history) is established for a structure being evaluated. To ensure that the variability in the response is addressed, a design response spectrum is then established using code guidance (e.g. ASCE 498 [1]). Because the design response spectrum does not match the response of the seed time history, the seed time history cannot be used for design evaluation. Instead, a modified time history must be used that is based on the seed time history, but provides a response defined by the design response spectrum. The method described in this paper is a way to modify the seed time history in such a way that it produces the design response.
There are other approaches to achieve the same purpose. One in particular is called RSPM [2]. RSPM [2] performs modifications on the seed acceleration using oscillator impulse response in reverse time order and a taper cosine wave. The primary seed acceleration modification technique used in the method described in this paper is to add low energy, tuned sine waves. RSPM [2] incorporates matrix inversion for simultaneous adjustments relative to differences between the seed and design response spectra. The method described in this paper makes adjustments at each natural frequency relative to the difference between the seed and design response spectra. Both methods are iterative. The primary motivation for pursuing the tuned sine wave method is the expectation of improved solver stability.

A more detailed description of the method described in this paper is as follows. This method is a multi-step process. It is accomplished with an initial scaling step and five refinement steps. While the method has been tested on many seed acceleration time histories and many design response spectra, this paper will use results from one example to demonstrate the method. The example is based on a reasonable seed acceleration time history with a time step of 0.005 seconds. The design response spectrum covers natural frequencies from $0.1 \mathrm{~Hz}$ to $100 \mathrm{~Hz}$ with 300 points evenly spaced on a log scale.

Figure 1 shows the seed acceleration time history and the corresponding seed velocity and seed displacement time histories derived using Fourier transform techniques. 

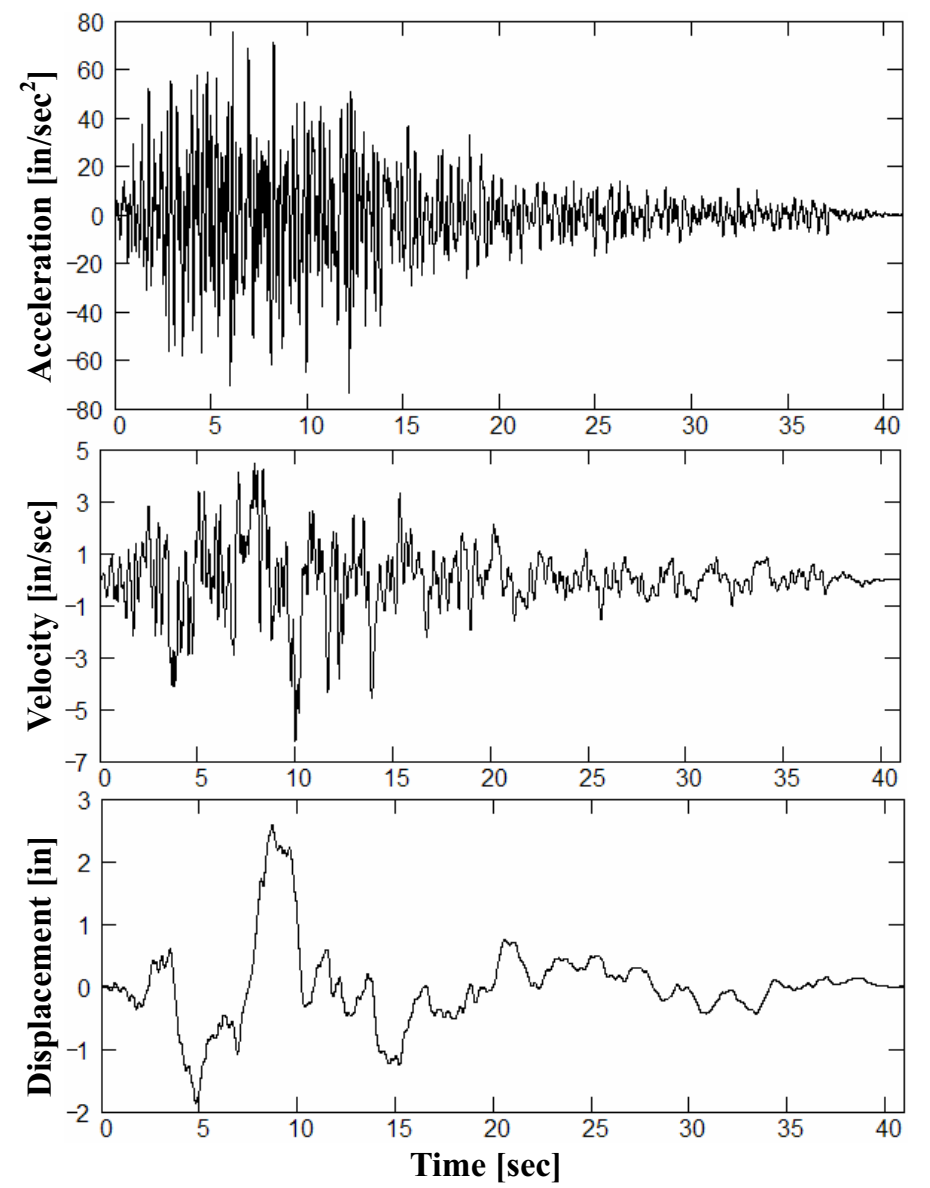

Figure 1. Seed motion time histories.

Figure 2 shows the response spectra for the acceleration, velocity, and displacement. The acceleration response spectra plot in Figure 2 also shows the acceptable error on the design response spectrum per ASCE/SEI 43-05 Section 2.4 [3]. Figure 3 shows the Fourier transform of the seed acceleration time history. Figure 4 is a trapezoidal rule evaluation of the seed acceleration to plot cumulative energy (Eq. 2.4-2 in ASCE 4-98 [1]). The plot shown is scaled to the final cumulative energy.
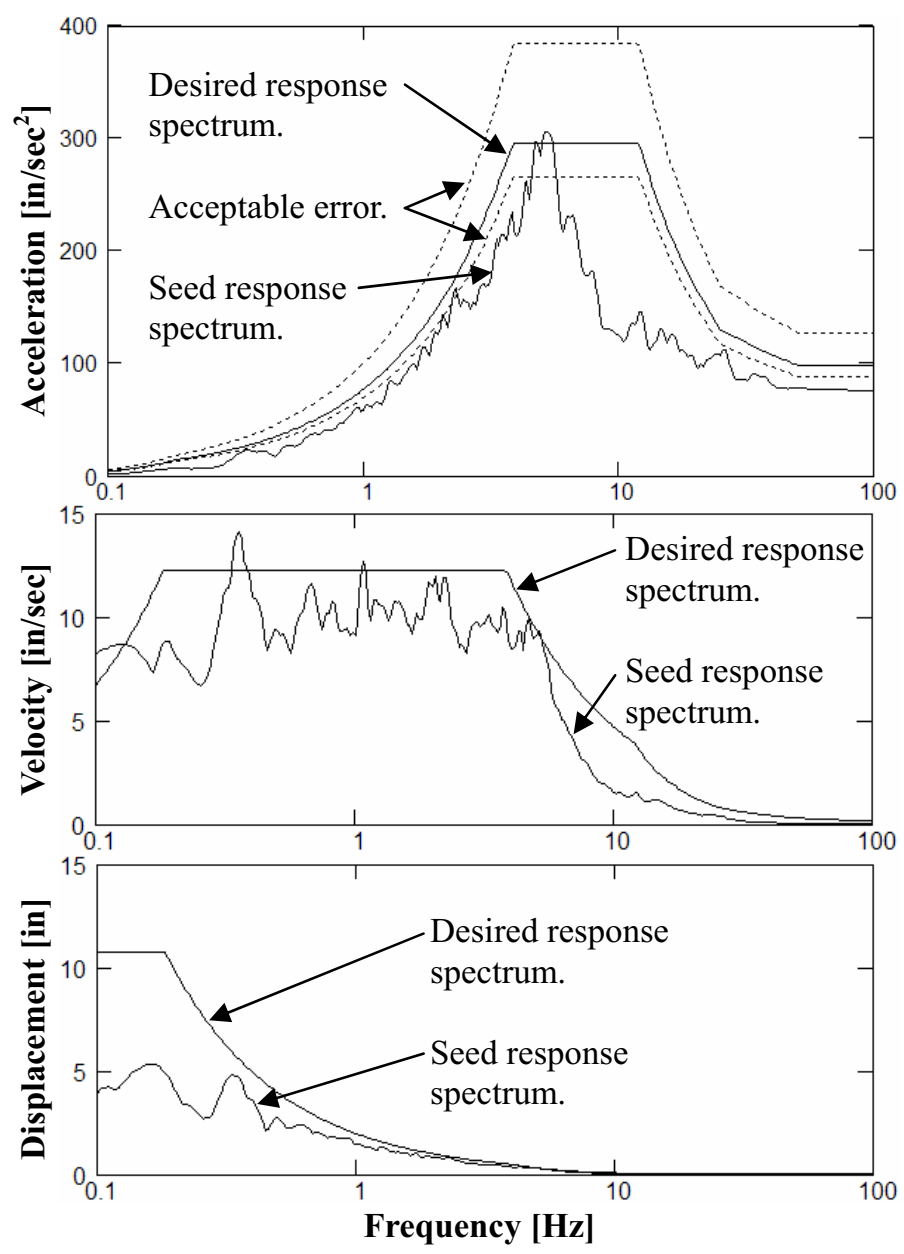

Figure 2. Seed and design response spectra.

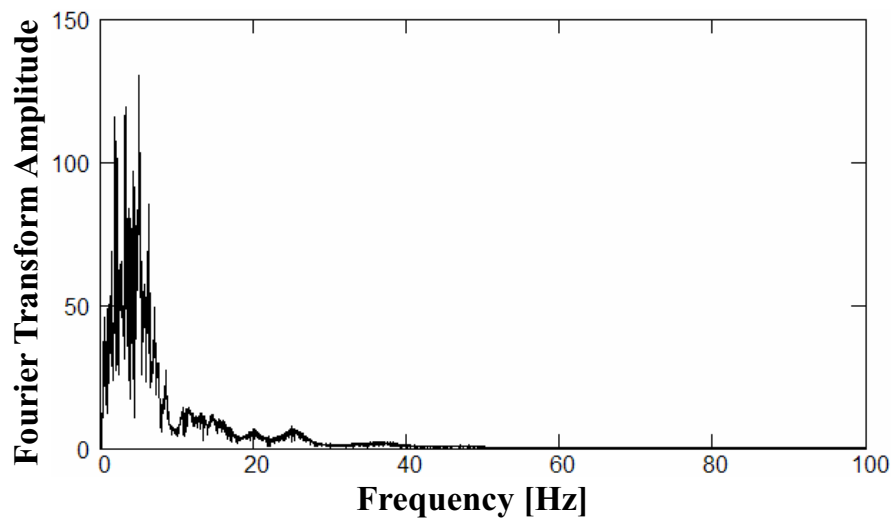

Figure 3. Fourier transform of seed acceleration. 


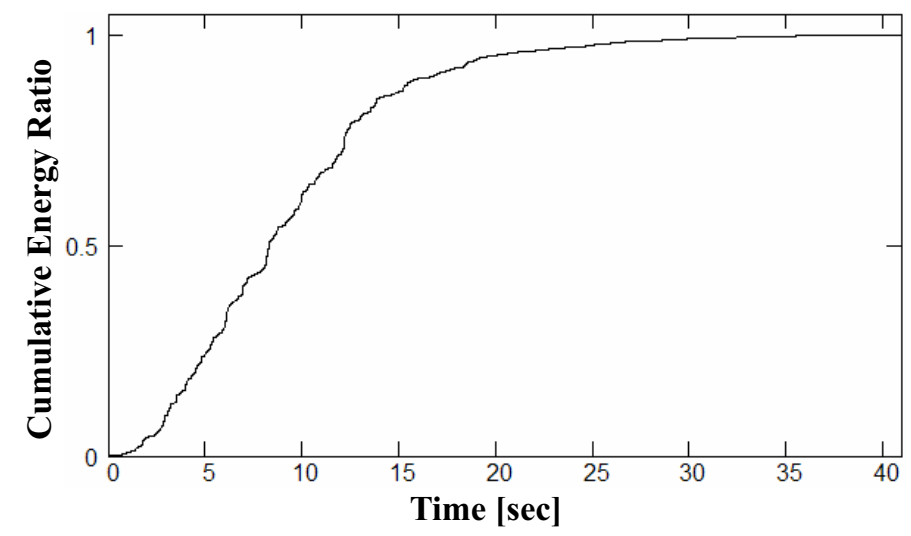

Figure 4. Seed cumulative energy ratio.

Many subroutines are used in the modification process. A few important ones are discussed here. First, there is an integrator subroutine that uses Fourier transform techniques along with an input acceleration time history to produce resulting velocity and displacement time histories. A second subroutine, also using Fourier transform techniques, is written that accepts an acceleration time history and produces the acceleration, velocity, and displacement response time histories for a simple damped oscillator. A third subroutine is written to use the previous two subroutines to produce acceleration, velocity, and displacement response spectra. It also includes data for the point in time when the peak acceleration values occur. Fourth and fifth subroutines are used to cause the velocity and displacement time histories to end with zero amplitude. Zeroing is performed after every acceleration time history modification to maintain control of drift. The fourth subroutine operates with a small linearly changing acceleration curve added to the acceleration time history. It is used where many iterations are necessary due to its relative stability. The fifth subroutine operates with smooth polynomial acceleration curves added to the start and end of the acceleration time history. It is used for intermediate steps due to its not affecting the middle region of the time history. Sixth, a subroutine is written that produces a smooth linear transition from one time history to another. This is used primarily to transition a modified time history back to previous time history at its start and end. (For example, consider a time span at the end of a modified time history to be transition back to a previous time history. At the start of the time span, the time history value is that of the modified time history. At a time $25 \%$ into the time span, the time history value is established by adding $75 \%$ of the modified time history value to $25 \%$ of the previous time history value. At the end of the time span the time history value is that of the previous time history.) A seventh subroutine is written to identify time history amplitude peaks. If the amplitude peaks are above some designated maximum, they are identified and if they are all below a designated minimum, the highest peak is identified. Then using a defined band of Fourier transform frequencies, a best fit adjustment time history is established to reduce the difference between the peaks and the desired amplitude. If the time history is not acceleration, conversion of the adjustment time history to acceleration takes place in the frequency domain. An eighth subroutine is written to compare a given time history to a target time history. The target time history is a time history with a general shape that is desirable, but an exact fit of it is not desirable. Then using a defined band of Fourier transform frequencies, a best fit adjustment time history is established to reduce the differences between the given time history and the target time history. If the time history is not acceleration, conversion of the adjustment time history to acceleration takes place in the frequency domain.

\section{INITIAL SCALING STEP}

The initial scaling step is meant to scale the Fourier transform amplitudes in such a way that the desired response is more closely matched. This consists of first developing a response array which can be used with the Fourier transform amplitudes to produce a response spectrum. At low frequencies there are more response spectrum points (based on the 100 points per frequency decade) than Fourier transform amplitudes. At high frequencies there are many more Fourier transform amplitudes than response spectrum points. Consequently, at low frequencies the amplitudes are scaled directly. However, at high frequencies where there are multiple Fourier transform amplitudes per response spectrum point, the response is scaled in two groups. The groups consist of those that scaling causes a positive change in the highest frequency response and those that scaling causes a negative change in the highest frequency response. Next, the response of the two groups is plotted with the design response. The two are then weighted based on how far above or below the design high frequency response they occur. They are recombined with the weighting and a change in acceleration time history is established. The average amplitude through time is then scaled relative to the seed average amplitude (to ensure a similar cumulative energy ratio curve) and the change in acceleration is added to the seed acceleration time history. The modified acceleration time history peaks are scaled with a $0 \mathrm{~Hz}$ to $50 \mathrm{~Hz}$ frequency band and the design amplitude is the $100 \mathrm{~Hz}$ value from the response spectrum. Then the modified acceleration time history is smoothly transitioned to the seed acceleration multiplied by the $100 \mathrm{~Hz}$ acceleration response spectrum value divided by the peak seed amplitude. The transition occurs such that at a time equal to zero the value is from the adjusted seed acceleration time history and at a cumulative energy value of $5 \%$ the value is entirely from the modified acceleration time history. Additionally, the transition occurs such that at a cumulative energy value of $95 \%$ the value is entirely from the modified acceleration time history and at a cumulative energy value of $99.9 \%$ and above the values are entirely from the adjusted seed acceleration time history. The resulting modified acceleration time history is then used to produce a displacement time history. A target displacement time history is also generated using the seed displacement time history multiplied by the ASC/SEI 43-05 [3] proportions. Scaling to the target 
time history is performed with a frequency band of $0 \mathrm{~Hz}$ to 0.5 $\mathrm{Hz}$. It is then converted to acceleration and used to modify the acceleration time history. Next, a subroutine is written that adjusts the velocity response for frequencies up to $5 \mathrm{~Hz}$. (The frequencies used are based on the 100 per decade but where this produces more frequencies than provided by the Fourier transform frequencies, just the Fourier transform frequencies are used.) It uses the velocity response and the design pseudo velocity response spectrum to perform a peak scaling operation. The frequency band used is the frequencies $15 \%$ above and below each frequency being modified. This is intended only to improve the low frequency velocity response, but this is the region where the improvement is most beneficial. The displacement from the resulting modified acceleration time history is again scaled to the previously defined target displacement time history, but only with a frequency band of 0 $\mathrm{Hz}$ to $0.09 \mathrm{~Hz}$. This only fixes gross distortions caused by the velocity peak scaling operation. Figure 5 shows the resultant acceleration response spectrum generated from the modified acceleration time history. The seed and design response spectra are also shown for comparison.

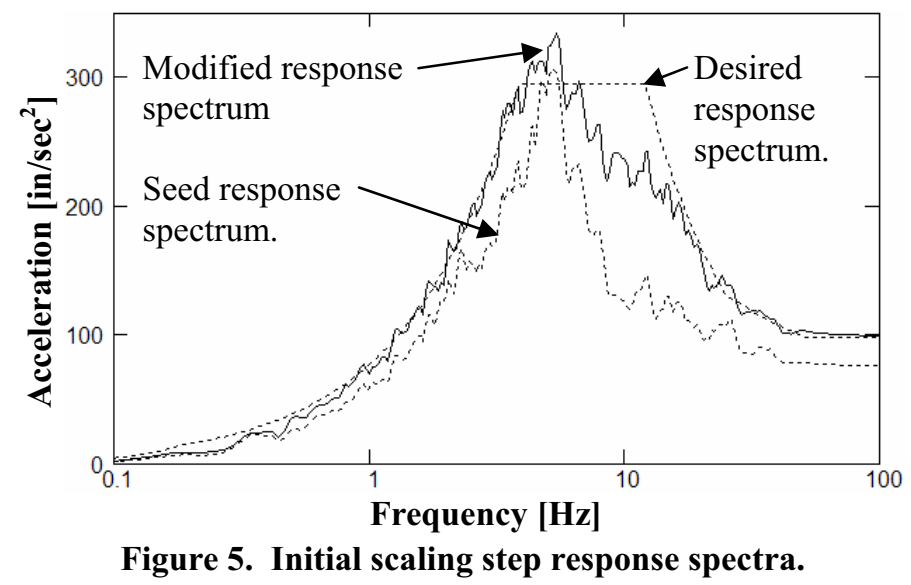

\section{SECOND STEP}

In the second step, time history adjustments are made to improve the low frequency response accuracy while adding minimal energy. Initially, a new target displacement is established. This is done by making a smooth transition from the final displacement time history of the first step to its corresponding target displacement time history. The transition is performed similar to that of the acceleration transition described in the first step. The resulting displacement time history is then multiplied by the ratio of the peak of the previous target displacement divided by the peak of the current target displacement. The second step acceleration adjustment is done by finding the peak response of a given natural frequency. The peak response is then adjusted to the design response by adding a sine wave tuned to best amplify the peak. The sine wave uses as many cycles as will fit in advance of the peak to minimize the required amplitude but not more than a user defined number. (The user defined number for the early adjustments is four and for the final adjustments it is eight.) Given that this step is for low frequency refinement, a displacement response comparison is performed up to about 3 Hz. The frequencies used are based on the 100 per decade but where this produces more frequencies than provided by the Fourier transform frequencies, just the Fourier transform frequencies are used. The modification process then includes starting at the high side of the frequency range and working down. As this occurs, several peaks are adjusted with sine waves going down in frequency. After each adjustment, the displacement is scaled to the target with a frequency band ending just lower than the lowest adjusted. At regular intervals the process is started back at the top and all of the previous frequencies are readjusted with a few extras added to the low end. Scaling back to the target only occurs with a frequency band ending just lower than the lowest adjusted over the whole process. Once modifications have occurred at all of the checked frequencies, a single pass of all the 100 per decade frequencies up to $3 \mathrm{~Hz}$ is performed. This is done with a target displacement time history of the modified displacement time history smoothed to the first target displacement time history. It is necessary for accuracy, but is relatively unstable so 5 additional passes with the first set of frequencies and the second target displacement time history are done to finish the sine wave modification. Then a third target displacement time history is created with the modified displacement time history smoothed to the first target displacement time history. The resulting modified acceleration time history is then $0 \mathrm{~Hz}$ to 50 $\mathrm{Hz}$ peak scaled to the $100 \mathrm{~Hz}$ response spectrum value. The modified acceleration time history is then adjusted by smoothing it to the acceleration time history in the first step at the point after smoothing was performed. To correct gross changes that could show up in the displacement time history, displacement scaling to the third target displacement is then performed with a $0 \mathrm{~Hz}$ to $0.09 \mathrm{~Hz}$ frequency band. Finally, 0 $\mathrm{Hz}$ to $0.2 \mathrm{~Hz}$ peak scaling is performed on the velocity and then displacement time histories to get them closer to the ASC/SEI 43-05 [3] proportions. Figure 6 shows the resultant acceleration response spectrum generated from the modified acceleration time history. The seed and design response spectra are also shown for comparison. 


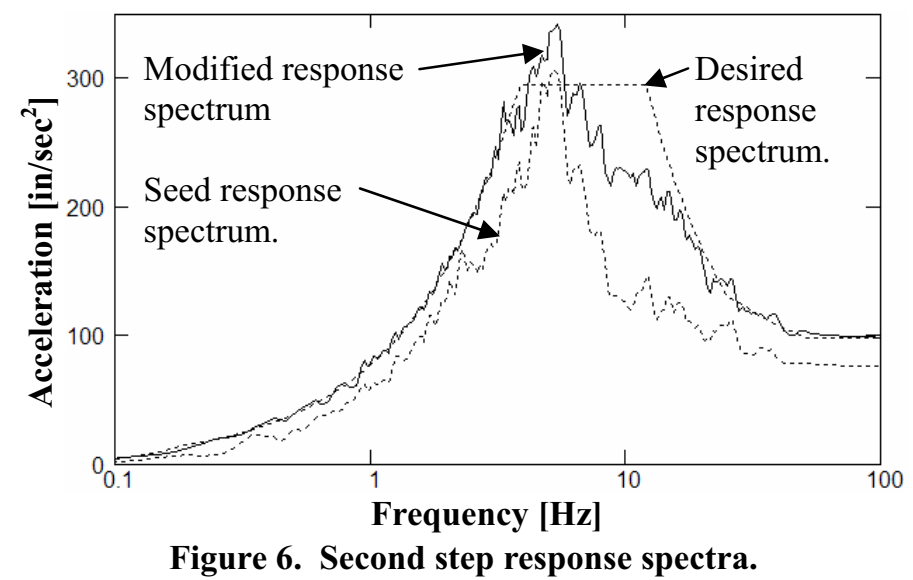

\section{THIRD STEP}

In the third step, time history adjustments are made to improve the high frequency accuracy of the response while adding minimal energy. Initially, a new target displacement is established by smoothing the final second step displacement to the first step target displacement. The third step acceleration adjustment is also done with the sine wave approach. Given that this step is for high frequency refinement, the acceleration response spectrum is used and the modification is performed from about $3 \mathrm{~Hz}$ to about $50 \mathrm{~Hz}$. The frequencies used are based on the 100 per decade. The modification process then includes starting at the low side of the frequency range and working up. As this occurs, ten peaks are adjusted with sine waves going up in frequency. After each adjustment, the displacement is scaled to the target with a frequency band ending at $0.9 \mathrm{~Hz}$. After each set of ten, the process is started back at the bottom and all of the previous frequencies are readjusted with ten extras added to the high end. The resulting modified acceleration time history is then $0 \mathrm{~Hz}$ to $50 \mathrm{~Hz}$ peak scaled to the $100 \mathrm{~Hz}$ response spectrum value. The modified acceleration time history is then adjusted by smoothing it to the acceleration time history in second step at the point after smoothing was performed. To correct gross changes that could show up in the displacement time history, displacement scaling to the current target displacement is then performed with a $0 \mathrm{~Hz}$ to $0.9 \mathrm{~Hz}$ frequency band. Figure 7 shows the resultant acceleration response spectrum generated from the modified acceleration time history. The seed and design response spectra are also shown for comparison.

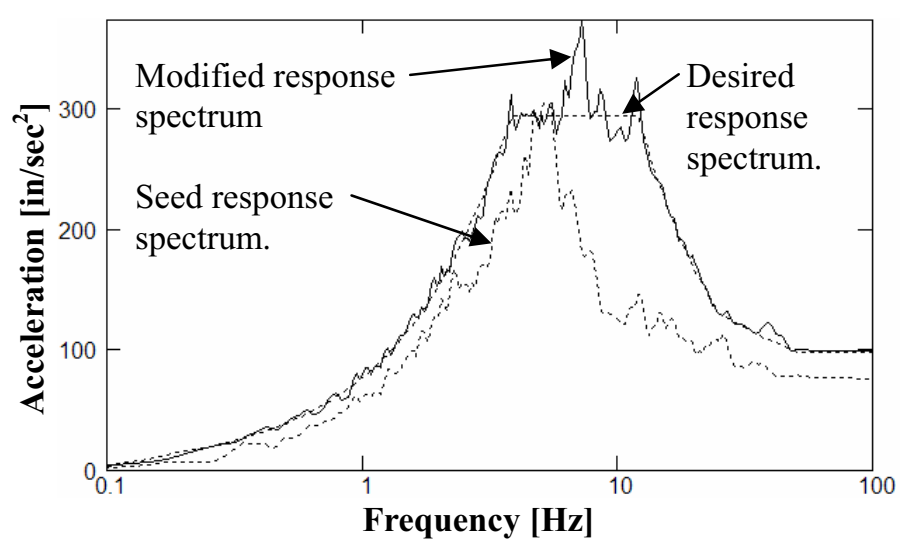

Figure 7. Third step response spectra.

\section{FOURTH STEP}

The second and third steps produce acceleration and displacement time histories that are close to the design. In the fourth step, the final low frequency time history adjustments are made. Initially, a new target displacement is established by smoothing the final third step displacement to the first step target displacement. The fourth step acceleration adjustment is done very similar to the first part of the second step. The primary differences are that the highest frequency is about $1 \mathrm{~Hz}$ and with each series of modifications, only one additional frequency is modified before the process is repeated. Afterward, five additional passes are done with the whole set of frequencies and the same target displacement time history. The resulting modified acceleration time history is then $0 \mathrm{~Hz}$ to 50 $\mathrm{Hz}$ peak scaled to the $100 \mathrm{~Hz}$ response spectrum value. The modified acceleration time history is then adjusted by smoothing it to the acceleration time history in third step at the point after smoothing was performed. To correct gross changes that could show up in the displacement time history, displacement scaling to the current target displacement is then performed with a $0 \mathrm{~Hz}$ to $0.09 \mathrm{~Hz}$ frequency band. Next, $0 \mathrm{~Hz}$ to $0.09 \mathrm{~Hz}$ peak scaling is performed on the velocity and then displacement time histories to get them closer to the ASC/SEI 43-05 [3] proportions. At this point, the resulting displacement time history is smoothed to the first step target displacement time history to create a second target displacement for this step. Then, $0 \mathrm{~Hz}$ to $0.5 \mathrm{~Hz}$ scaling is performed to the second target displacement time history. The result is then put through five additional sine wave modification passes done with the whole set of frequencies for this step and the second target displacement time history. Next, the acceleration time history end is smoothed to the previously smoothed acceleration time history in this step and the initial value is reset to zero. At this point, the end of the resulting displacement time history is smoothed to the first step target displacement time history to create a third target displacement for this step. Then, $0 \mathrm{~Hz}$ to $0.5 \mathrm{~Hz}$ scaling is performed to the third target displacement time history. Finally, the start and end of the acceleration curve, which should be very close to zero amplitude are enforced to be zero. Figure 8 shows the resultant acceleration 
response spectrum generated from the modified acceleration time history. The seed and design response spectra are also shown for comparison.

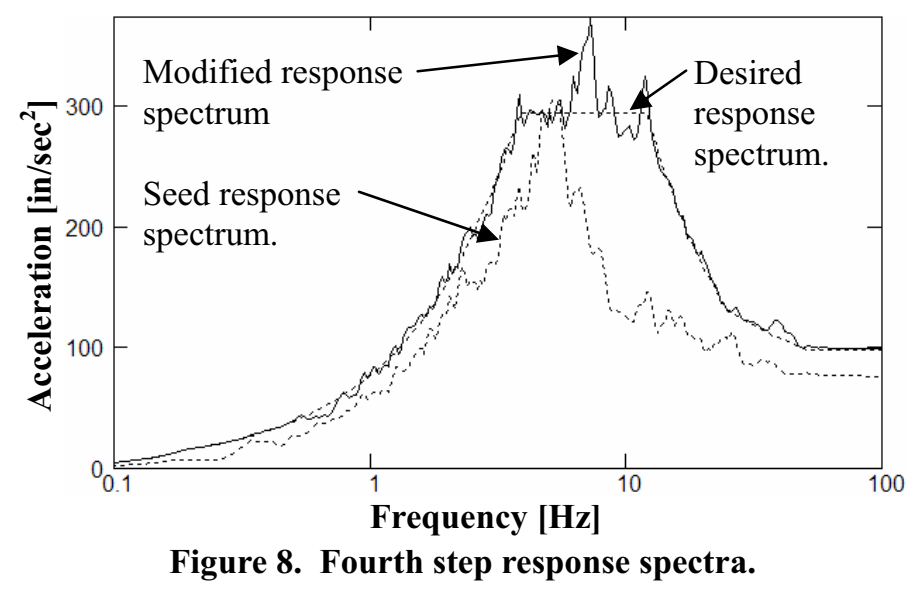

\section{FIFTH STEP}

In the fifth step, time history adjustments are again made to improve the high frequency accuracy of the response while adding minimal energy. Initially, a new target displacement is established as the end of the fourth step displacement time history. The fifth step acceleration adjustment is done similar to the third step except the frequency ranges is from about 0.5 $\mathrm{Hz}$ to about $50 \mathrm{~Hz}$. Also, the modification process is done by sweeping the entire frequency range from low to high fifty times. After each adjustment, the displacement is scaled to the target with a frequency band ending at $0.5 \mathrm{~Hz}$. The resulting modified acceleration time history is then adjusted by smoothing it to the final acceleration time history in the fourth step. To correct gross changes that could show up in the displacement time history, displacement scaling to the current target displacement is then performed with a $0 \mathrm{~Hz}$ to $0.5 \mathrm{~Hz}$ frequency band. Figure 9 shows the resultant acceleration response spectrum generated from the modified acceleration time history. The seed and design response spectra are also shown for comparison.

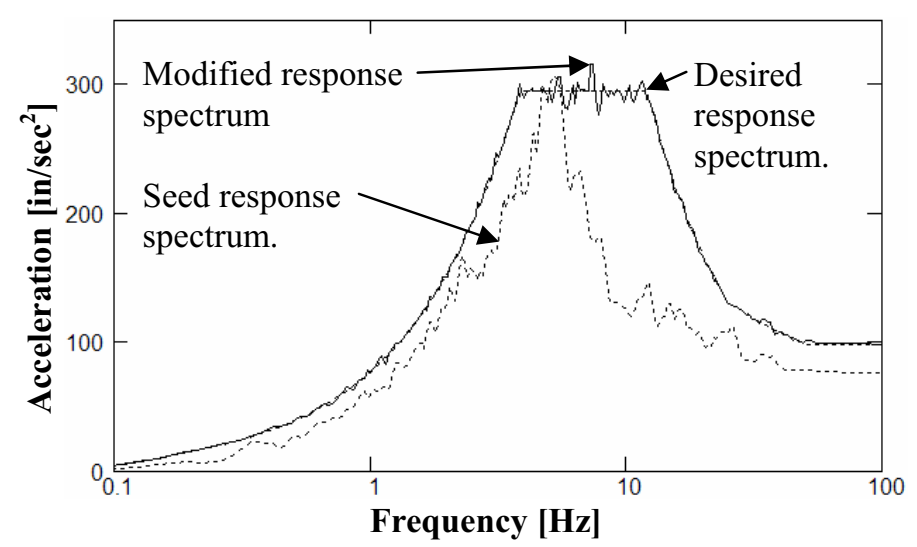

Figure 9. Fifth step response spectra.

\section{SIXTH STEP}

In the sixth step, final time history adjustments are made to the high frequency response. This acceleration adjustment is done similar to the third step except the frequency ranges is from about $0.25 \mathrm{~Hz}$ to $100 \mathrm{~Hz}$. Also, the modification process is done by sweeping the entire frequency range from low to high thirty-five times. After each adjustment, the displacement is scaled to the target with a frequency band ending at $0.25 \mathrm{~Hz}$. The resulting modified acceleration time history is then adjusted by smoothing it to the final acceleration time history in the fourth step. To correct gross changes that could show up in the displacement time history, displacement scaling to the fourth step target displacement is then performed with a $0 \mathrm{~Hz}$ to $0.25 \mathrm{~Hz}$ frequency band. At this point, several minor cosmetic modifications are performed. First, the first tenth of a second of the modified acceleration time history is smoothed back to the final acceleration time history in the fourth step. Second, the last one percent of the time relative to energy for the modified acceleration time history is smoothed back to the final acceleration time history in the fourth step. (These are done separately to improve the accuracy of the zeroing subroutines.) Finally, the start and end of the acceleration time history are zeroed. (This is repeated twice because the zeroing of the velocity and displacement time histories can change the start and end of the acceleration time history by a very small amount.) This results in acceleration, velocity, and displacement time histories that begin and end at zero. They also produce accurate acceleration and displacement response spectra (and reasonable pseudo velocity response spectrum results). Figures $10-14$ show the final results along with the data from Figures $1-4$. Figure 14 shows the power spectral density only up to $20 \mathrm{~Hz}$. As demonstrated by Figure 12, both the original and modified curves become less interesting for frequencies from $20 \mathrm{~Hz}$ to $100 \mathrm{~Hz}$. 


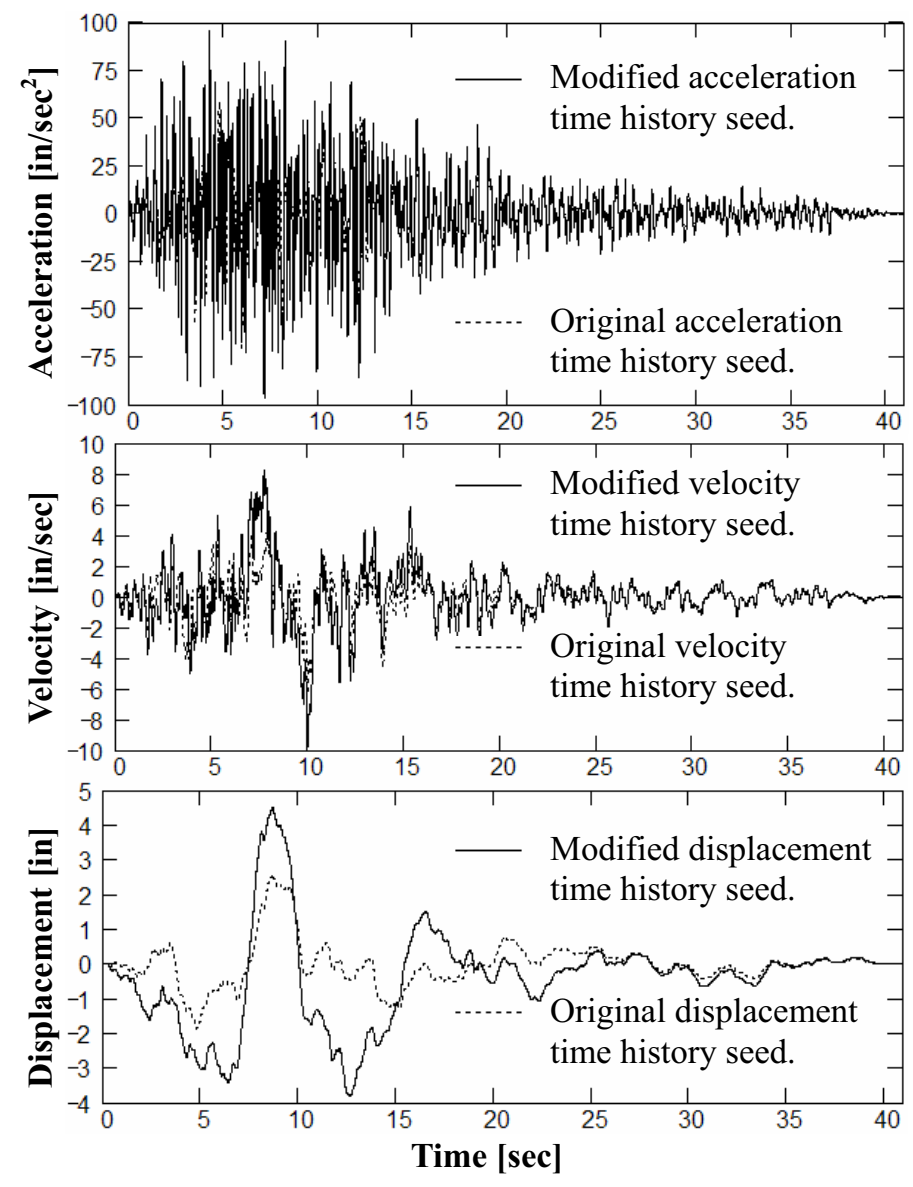

Figure 10. Motion time histories.
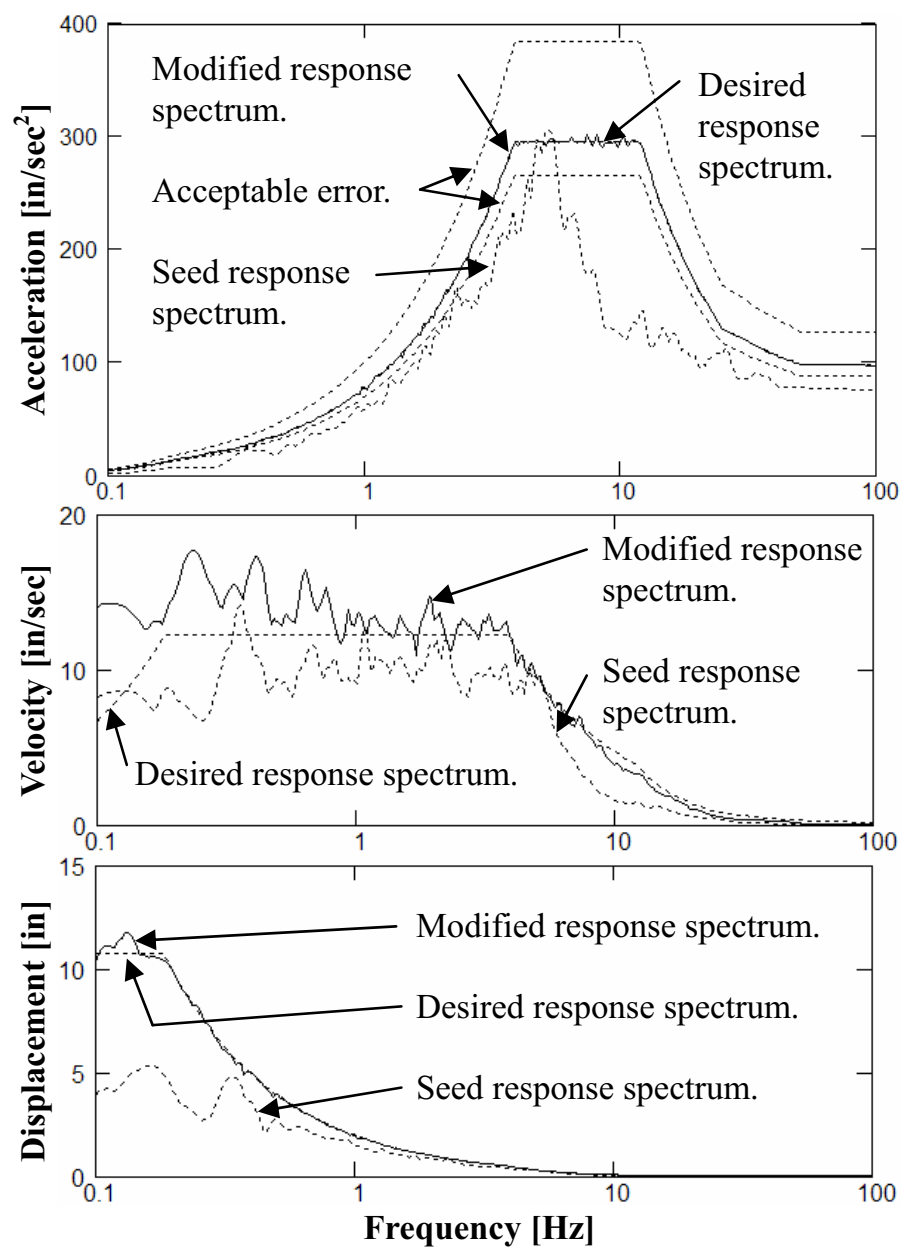

Figure 11. Response spectra.

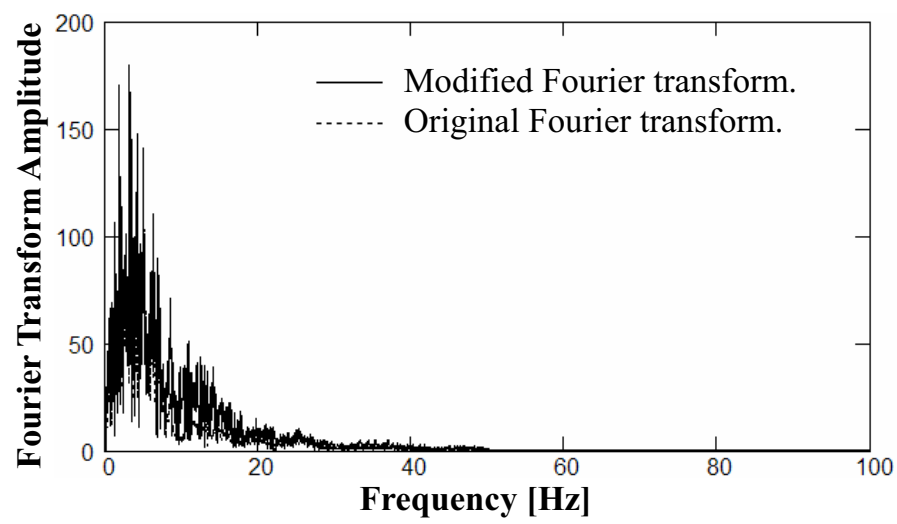

Figure 12. Fourier transforms relative to acceleration. 


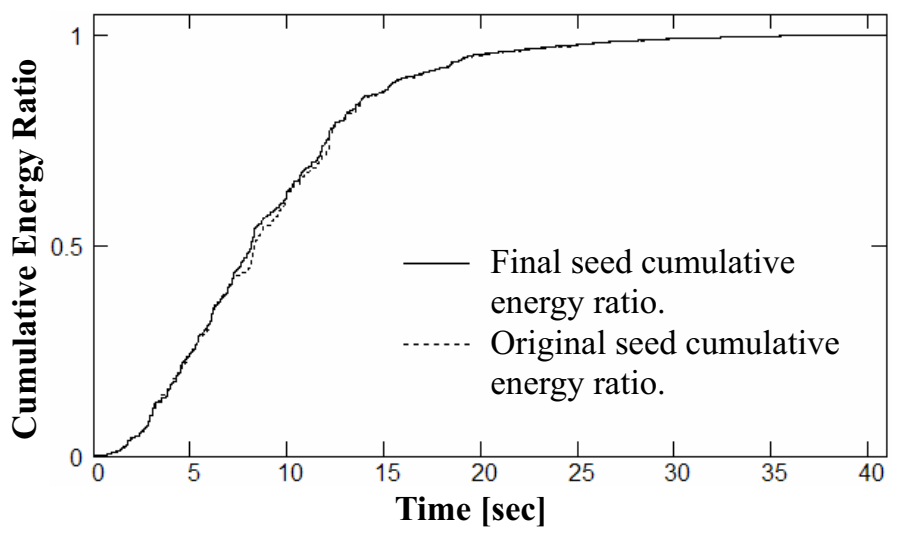

Figure 13. Cumulative energy ratios.

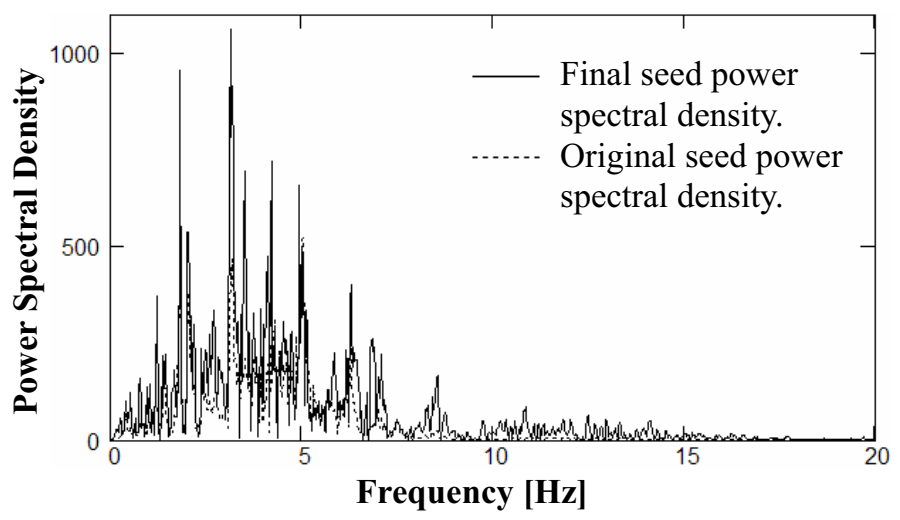

Figure 14. Power spectral densities.

\section{CONCLUSION}

A method has been developed which takes a seed earthquake time history and modifies it to produce given design response spectra. It performs in a stable manner that requires no matrix inversion. It encourages the motion time histories to meet the code acceleration, velocity, and displacement ratios and attempts to fit the pseudo velocity response spectrum. Additionally, the acceleration, velocity, and displacement time histories are modified to ensure that they start and end with an amplitude of zero.

The subroutines described in this paper were programmed in Mathcad along with many additional calculations and plots used for checking when the method was being developed. The model described in the paper took several hours to run in this form on a computer with a $2.00 \mathrm{GHz}$ processor. The expectation is that a streamlined version programmed in $\mathrm{C}$ could produce results for a problem using similar parameters in less than an hour.

\section{ACKNOWLEDGEMENTS}

This manuscript has been authored by Battelle Energy Alliance, LLC under Contract No. DE-AC07-05ID14517 with the U.S. Department of Energy. The United States Government retains and the publisher, by accepting the article for publication, acknowledges that the United States Government retains a nonexclusive, paid-up, irrevocable, world-wide license to publish or reproduce the published form of this manuscript, or allow others to do so, for United States Government purposes.

\section{REFERENCES}

[1] ASCE 4-98, Seismic Analysis of Safety-Related Nuclear Structures and Commentary, American Society of Civil Engineers, 1998.

[2] Validation Manual, RSPM, Version $02 \mathrm{w} /$ Amendments, June, 262002.

[3] ASCE/SEI 43-05, Seismic Design Criteria for Structures, Systems, and Components in Nuclear Facilities, 2005. 